\title{
Ancient Schwannoma of Vocal cord
}

\author{
Anuj Khurana ${ }^{1}$ and Nayana Patil ${ }^{2 *}$ \\ 'Dept. of Histopathology, Max Super Speciality Hospital, Saket, New Delhi., India \\ ${ }^{2}$ Dept. of Pathology, Max Super Speciality Hospital, Saket, New Delhi. India
}

\section{Dear Sir,}

We discuss a case of a 54 year old male who presented to ENT department of our hospital with complaints of hoarseness of voice. Otorhinolaryngological examination with indirect laryngoscopy showed a polypoidal mass arising from the left vocal cord. A micro laryngeal surgical excision was planned. Intraoperative findings confirmed a polyp arising from the left vocal cord, which was firm, vascular, restricting the motility of vocal cord. Opposite cord was normal and mobile. The resected tissue was sent for routine histopathology. Gross examination revealed an encapsulated mass measuring $2.5 \times 1.5 \times 1.5 \mathrm{~cm}$, which on cut section revealed homogeneous pale yellow myxoid surface [Figure 1]. Microscopic examination showed surface lining of nonkeratinized stratified squamous epithelium ulcerated by a circumscribed spindle cell tumor with hypo and hypercellular areas [Figure 2]. The latter comprising of short and long fascicles with a verocoid arrangement focally [Figure 3]. At places the cellular areas showed features of nuclear enlargement, hyperchromasia and bizarre nuclei. Hypocellular areas showed myxoid changes, hyalinised blood vessels were also noted [Figure 4]. There was no evidence of increased mitosis or necrosis. A morphological diagnosis

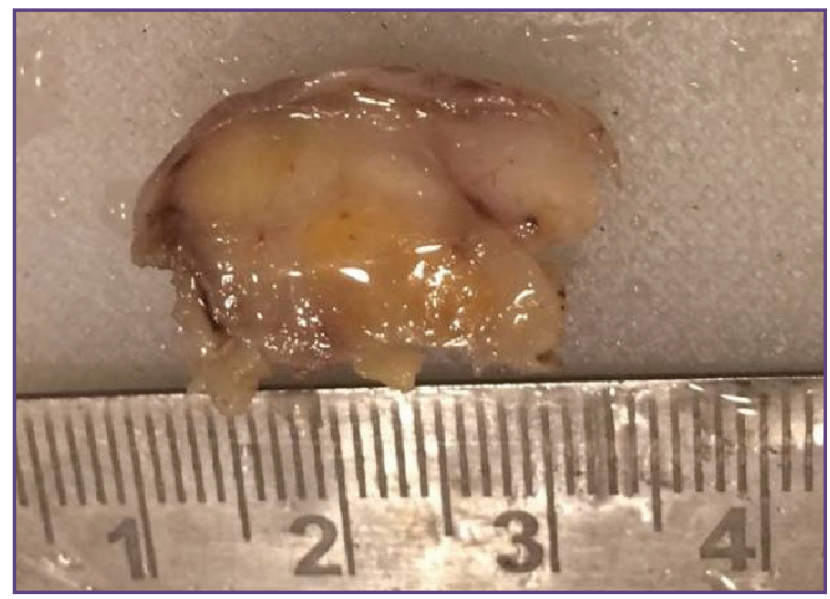

Fig. 1: Gross photograph reveals cut section of the polypoidal mass with a myxoid appearance. of a nerve sheath tumor was considered. Corroboratory immunohistochemistry was performed.

The lesional cells showed strong nuclear positivity for S-100 protein [Figure 5]. They were expectedly immunonegative for Pancytokeratin(CK). Ki 67 proliferative index was low $(<01 \%)$.A final diagnosis of schwannoma with ancient changes was rendered. The postoperative course was uneventful, and at routine follow-up, the patient was symptom-free.

Vocal cord schwannoma is a rare phenomenon. They represent 0.1 to $1.5 \%$ of all benign tumors of larynx,with $80 \%$ arising from aryepiglottic fold, $20 \%$ from false or true vocal cords. They normally present as a slow-growing, encapsulated, submucosal mass. ${ }^{[1]}$ Vocal cord schwannomas can present with hoarseness, dyspnea, dysphagia, stridor. There is one case reported in literature of death due to asphyxia. ${ }^{[2]}$

Laryngeal schwannoma are thought to arise from schwann cells of internal branch of superior laryngeal nerve.$^{[3]}$ It is a benign nerve sheath tumor, which requires distinction from neurofibroma as the latter is closely intertwined with nerve fibers and hence difficult to excise completely leading to its recurrence. The distinction between the two entities is to be emphasized as malignant transformation occurs in $10 \%$ of neurofibromas, but very rare in schwannomas. ${ }^{[4]}$

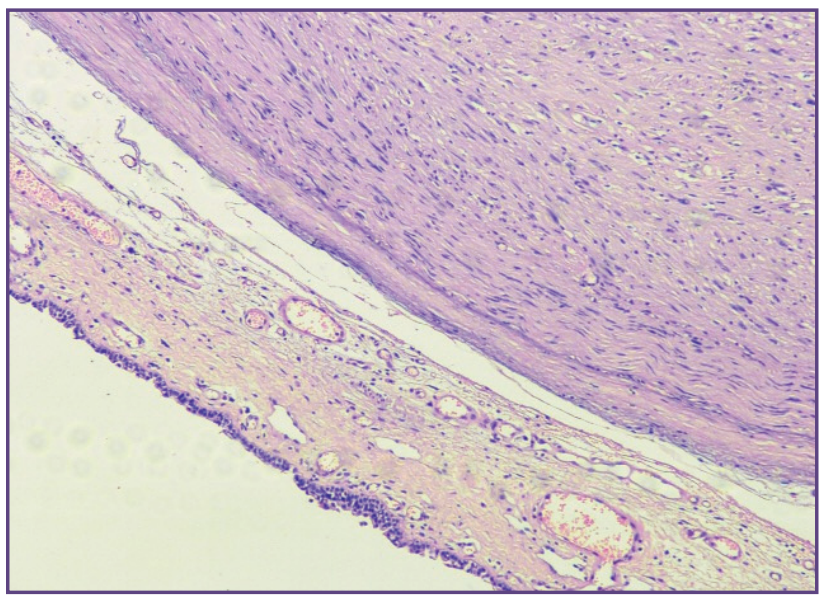

Fig. 2: Microphotograph showing a circumscribed spindle cell neoplasm; the overlying epithelium is unremarkable. (H and $E, \times 100$ ). 


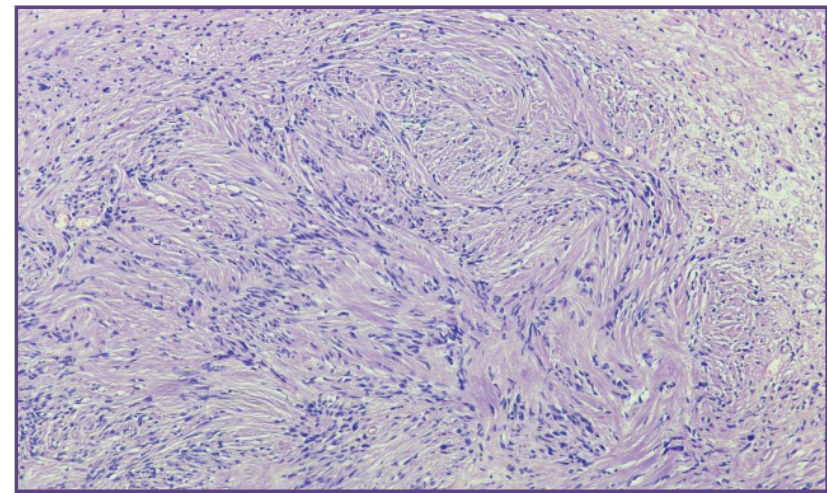

Fig. 3: Microphotograph showing cellular spindle cell proliferation with architecture reminiscent of verocay bodies. ( $\mathrm{H}$ and $\mathrm{E}, \mathrm{x} 400$ ).

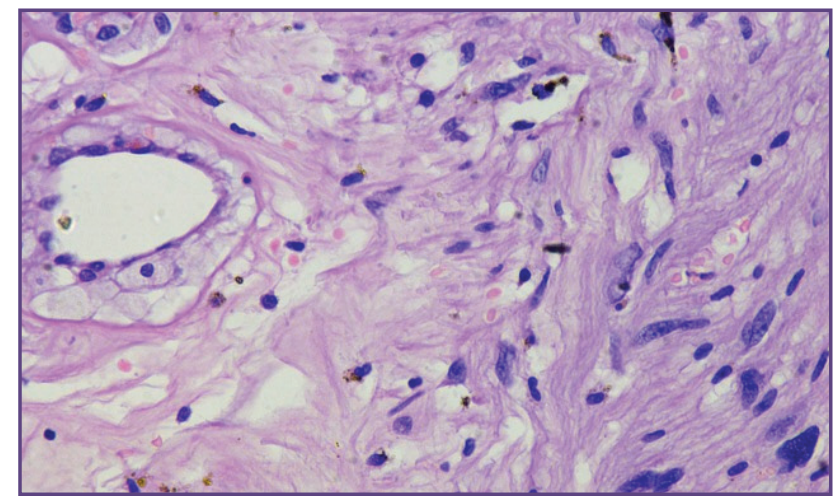

Fig. 4: Microphotograph showing ancient changes in the spindled areas with hypocellular areas showing foamy macrophages. ( $\mathrm{H}$ and $\mathrm{E}, \times 400)$.

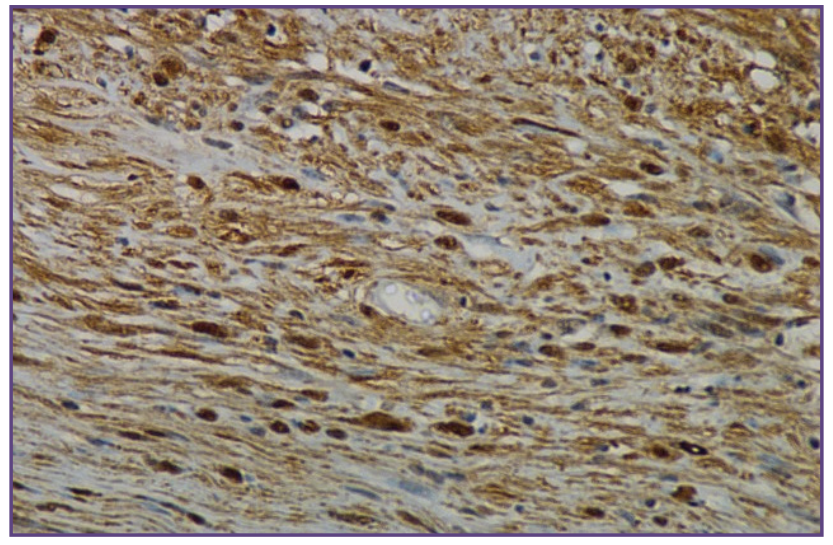

Fig. 5: Immunohistochemistry showing S-100 nuclear expression $(\mathrm{DAB}, \times 400)$.

Vocal cord schwannoma of ancient variety is essentially a benign entity characterized by bizzare changes in the form of significant nuclear atypia or pleomorphism. ${ }^{[5]}$

To conclude, we present a case of vocal cord schwannoma which remains largely unsuspected by the clinician owing to its rarity. Diagnostic interpretation of these features as a spectrum of degeneration becomes relevant to prevent a false overdiagnosis of a pleomorphic sarcoma; especially in small biopsies where in a complete excision is not performed.

\section{Reference}

1. Nalluri K, Palukuri S. Vocal Cord Schwannoma - A Rare
Case. International Journal of Recent Trends in Science and Technology. 2014; 9: 436-7.

2. Chiu CC, Chou SH, Wu CC, Liang PI, Lee KW. Obstructive laryngeal schwannoma in a young female. Chiu et al. World Journal of Surgical Oncology 2015; 13:24.

3. Chandrashekhara S H, Bhala A S, Kartikeyan K V, Shukla B, Safaya R. Vocal Cord Schwannoma - A Rare Case Report. J Can Res Ther. 2010; 6:543-5.

4. Taylor J, Stiefel M, Park SY. Schwannoma of the true vocal fold: a rare diagnosis.Ear Nose Throat J. 2006;85:52-3, 59.

5. Fletcher C. D., Peripheral neuroectodermal tumors. In: Fletcher C.D,.editor. Diagnostic histopathology of tumors. 3rd ed. Boston: Elsevier 2007.

*Corresponding author:

Dr. Nayana Patil, Department of Lab Medicine, Max Super Speciality Hospital, Saket, New Delhi- 110017, INDIA

Phone: +91 9560361064

Email: dr.nayana04@gmail.com

Financial or other Competing Interests: None.

Date of Submission : 12.10.2016

Date of Acceptance : 19.11.2016

Date of Publication : 19.02.2017 